\title{
An Approach to Systematic Part Design
}

\author{
Horea T. Ilies Vadim Shapiro \\ Department of Mechanical Engineering \\ 1513 University Avenue \\ University of Wisconsin-Madison, 53706 USA \\ Email:vshapiro@engr.wisc.edu
}

\begin{abstract}
The rise of solid modeling as a principal medium for mechanical product description can be traced to the requirement of informational completeness of geometric representations. Unfortunately, traditional geometry-based systems do not contain important information needed for many engineering activities and tend to force costly iterations in a product development cycle. We seek to understand spatial properties and combinatorial structure of mechanical parts in terms of simple interacting constructs related to part functionality and manufacturing processes. Existence of such a structure is illustrated for a simple part that is characterized in terms of its kinematics, strength, spatial containment, and manufacturing characteristics. The resulting explicit representation of correspondence between mechanical characteristics and spatial elements allows systematic product development, efficient modification procedures, and rational tolerancing approaches - eliminating many costly errors and iterations in a product development cycle. The study also highlights the lack of and the need for formal models supporting systematic design process.
\end{abstract}




\section{GEOMETRIC MODELS IN MECHANICAL DESIGN}

\subsection{Historical perspective}

It is a standard practice to describe mechanical devices and processes using the language of geometry to support all engineering activities, including conception, detailed design, tooling, manufacturing, inspection, assembly, and so on. Historically, this practice is well justified because shape is relatively easy to visualize and describe using graphical (drawing) methods, while mathematical models of mechanical function and physical processes are seldom known and/or understood by practicing engineers to the extent that they can provide means for unambiguous communication. * Until the second part of this century, engineering drawings also contained a variety of symbolic and textual information related to functionality and manufacture of the specified device, such as tolerances, record of changes, material properties, process characteristics, standard components, and so on (Booker 1979, chapters 15-16).

More recently, many engineering standards specify that non-geometric information (and in particular, information related to manufacturing methods) should not appear on the drawings (ANSI Standard Y14.5M). One common justification for this practice is the principle of process independence that tend to facilitate outsourcing and greater flexibility in choosing manufacturing methods. This approach to production of mechanical artifacts is consistent with the modern view that mechanical 'form' is induced from 'function,' and 'fabrication' follows 'form' (Rinderle 1986, Voelcker 1988). The separation of process and functional information from mechanical form had also a number of other important consequences:

- drafting, descriptive geometry, and later geometric modeling and computer-aided design, have become a specialized engineering skill;

- missing non-geometric information led to poor communication between design and manufacturing departments (accompanied by long lead-times, many iterations, and high costs); and

- it became easy to "design" geometric shapes that are difficult or impossible to manufacture.

This dominance of geometric information resulted in the emergence of new disciplines such as 'design for $\mathrm{X}$,' 'manufacturability analysis,' 'feature-based design and manufacturing,' and so on - all are built on the premise that 'geometric engineering' provides a firm rigorous foundation for all engineering activities. This view has been further supported by the rise of solid modeling notions and systems as a principal medium for mechanical product description, which in turn can be traced to the requirement of informational completeness of computer representations (Requicha \& Voelcker 1981). But it should be emphasized that a solid model is informationally complete in a very precise and restricted sense: under the assumption of unambiguous point membership classification, ${ }^{\dagger}$ a solid model contains information that is in principle sufficient for computing any geometric property of the nominal solid. In this sense, solid models of mechanical parts have become the smallest valid informational entities.

Nominal solid models appear to reflect many important characteristics of mechanical artifacts (Requicha \& Voelcker 1981). However, the notion of geometric information completeness does not easily

\footnotetext{
*Both descriptive geometry and modern physics are based on developments in the 16-17's century, but elements of engineering graphics have been practiced by Greeks and perhaps earlier (Booker 1979, Dimar 1993).

†Point Membership Classification is a procedure for deciding whether a given point is in, on, or outide of a solid model (Tilove 1980).
} 
extend to other physical and engineering characteristics of mechanical products. What is 'physical' has not been resolved even within the geometric modeling community; increasingly, it is argued that many mechanical objects should be modeled as more general point sets (Weiler 1986, Rossignac \& O'Connor 1989, Gursoz, Choi \& Prinz 1990).

\subsection{The current state of technology}

Form modeling The desire to capture and communicate more engineering information than 'just geometry' is partially responsible for the rise of so-called "feature-based" modeling systems. While it is universally agreed that features are useful for solid modeling, and many informal descriptions of features have been put forward, no formal definition of features exists (Rossignac 1990, Pratt 1988, Cunningham \& van Dixon 1988, Shah 1991). Perhaps most importantly, features are intended to capture and represent engineering "knowledge" and "function," but there are no agreed means for doing so, and features do not explicitly include any physical information. Sometimes, it may be convenient to think about features as named and typed (geometric) information structures that have to be combined with function and fabrication models - when and if such models become available.

Today, the major advantage of solid modeling systems (and feature-based systems in particular) lies in enormous increases in productivity of the creation and manipulation of geometric data, even if engineering paper drawings are still usually produced from that data. Geometric models support a variety of spatial computations, but they are not equipped to explicitly represent more general physical phenomena, such as heat transfer, stresses, or magnetic flux. Such quantities are simply not part of the assumed mathematical model.

Mechanical function An important approach to modeling of mechanical systems grew out of the apparent similarities between electrical circuits and other kinds of physical systems (see a historical survey by Evans and Dixhoorn (Evans \& van Dixhoorn 1974). One network-based formalism, the bondgraph, was developed by Henry Paynter (Paynter 1961). Using this methodology, a physical system is "reticulated" into a bond-graph by systematic "lumping" of spatially distributed physical quantities, so that the system's dynamic behavior can be simulated and analyzed.

A number of researchers have used bond graphs to model and represent the structure of mechanical systems for the purposes of design (as opposed to analysis). Notably, Finger and Rinderle (Finger \& Rinderle 1981) suggested formulating mechanical design procedures as function-preserving transformations on bond graph models. A bond graph is an example of a more general class of graph-based representation that describe the mechanical functionality and form in qualitative terms and schematically (Shah 1988, Ulrich 1989, Duffey \& Dixon 1988). A novel approach to design using schematic descriptions was proposed by Ulrich, who has identified the problem of 'function sharing' and developed heuristic methods to deal with it (Ulrich 1990) $\ddagger$

Graph-based and schematic representations are capable of capturing some interaction of mechanical form and function; but they do not deal with spatial distributions of material and other physical quantities, which are the distinguishing characteristics of mechanical systems when compared, say, to electrical networks. A combinatorial nature and several principles of the spatial form-function relationship has been observed in (Shapiro \& Voelcker 1989); Shimada and Gossard (Shimada \& Gossard 1992) used these principles to partially automate the design of mechanical components. Many other researchers have attempted to integrate geometric and functional information with varying degree of success (Finger \&

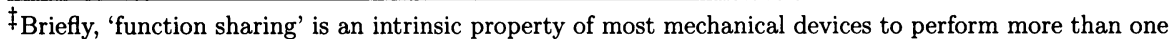
function in the same physical medium.
} 
Dixon 1989). Most known results use heuristics as an important component of form-function interaction, and the observation made by Voelcker in (Voelcker 1988) is still true today: "One of the major gaps in the understanding of design is the lack of means for modeling mechanical "function" in a manner that links function to form." Because of that, synthesis (the process of inducing form from function) is generally viewed as a poorly understood creative activity performed by humans (Ferguson 1993).

Form and fabrication In principle, manufacturing processes can be modeled as mathematical problems of physics, but models of real processes are quite complex, and they offer little insight in selecting a manufacturable form a priori. Because of this, much of manufacturing information is dominated by rules and tables that tend to divide all manufacturing process plans into a finite number of categories, based on factors like tolerances, material types, gross dimensions, presence or absence of certain features, material thickness, and production volume (Bralla 1986). Thus, injection molded and die cast parts are usually classified into several groups according to the expensive tooling they may require (Poli \& Shanmugasundaram 1991); machined parts into several tolerance classes (Bralla 1986), and so on.

Such process models provide the basis for various 'design for $\mathrm{X}$ ' and design rating paradigms (National Research Council 1990). But it can be shown (Peters, Rosen \& Shapiro 1994) that such models of formfabrication interaction can be incompatible with parametric definitions of functional forms. For example, traditional manufacturing methods are often dominated by tooling activities, and small changes in design parameters can easily lead to large changes in the tooling costs that are difficult to predict or control.

\subsection{Missing structure}

Despite the progress in feature-based modeling, identifying a reasonable combinatorial structure of mechanical artifacts has remained an elusive goal for a number of reasons, notably due to the intrinsically spatially distributed nature of mechanical continuum and multiplicity of functions supported by the same object. To illustrate the existence of such a structure, we will show how a simple automotive part, a secondary hood latch, can be characterized in terms of its kinematics, strength, spatial containment, and manufacturing characteristics. We show that each of these primitive characteristics partially defines the shape of the part and explore feasibility of systematically combining these elementary shape elements to obtain a single class of parts with common characteristics.

It appears that solid modeling may not adequately support many engineering activities, including systematic product design, maintenance of engineering changes, manufacturability analysis, tolerancing, cost optimization, and others. In particular:

- many engineering activities require a much finer level of representation than it is provided by solid modeling;

- important functional and manufacturing information is not properly integrated with geometry-based systems, and is rarely represented anywhere;

- the false perception of 'informational completeness' often leads to an iterative (and inefficient) product development cycle.

Our study shows that new formal models and representations of mechanical parts are needed for proper support of product development cycle. Such models must combine combinatorial, physical, and geometric information in manner that is consistent with solid modeling technology. New computer representations must include partially specified geometry, functional, and manufacturing information represented in a hierarchical fashion. 


\section{EXAMPLE: STUDY OF AN AUTOMOTIVE HOOD LATCH}

Figure 1 shows the solid model of a secondary hood latch, which is part of a typical hood latch assembly. It is a relatively simple, essentially two-dimensional part that nevertheless exhibits a fairly rich structure for the purposes of our study.

The functional purpose of a hood latch assembly is to engage and retain a (generally cylindrical) striker, which is attached to the hood of the automobile, and to prevent it from accidental release. This is usually accomplished by two different components: a primary latch which retains the striker, and a secondary latch which prevents it from accidental release. Our study will focus on the secondary latch.

During its downward motion, a vertically moving striker forces the secondary latch to rotate, as indicated in Figure 2(a), and latch it. The range of this rotation is limited by surface D (see Figure 2(c)) and mostly restricted by packaging constraints. While the striker continues to move vertically down, the secondary latch is brought back to its original position by the attached reaction spring (Figure 1); the primary latch, not shown, closes down and holds the striker fixed. The release of the striker from the hood latch assembly is accomplished by applying the required forces by means of handles: first indirectly to the primary latch from the cabin, and then directly to the secondary latch - so that it rotates and the hood can be lifted. The spring attached to the secondary latch provides the reaction force needed to insure its correct return to its original position, but the latch must continue to function even if the spring breaks. The secondary latch must also prevent the upward motion of the striker (and therefore of the hood) in case the primary latch fails to engage the striker or is accidentally released.

In what follows, we analyze the designed geometric form of the latch in terms of:

- Kinematics - geometry related to latch interactions with other moving parts;

Strength - the ability to withstand the specified loads;

Spatial containment - non-interference with other parts;

Manufacturing - process related considerations.

In this particular case, these categories largely determine the shape of the latch, but in general this list need not be complete, and there may be a number of other important considerations, such as weight, aesthetics, and so on. For simplicity, the following discussion focuses on the two-dimensional profile of the secondary latch, but generalizations to the full three-dimensional model are straightforward.

\subsection{Kinematic functionality}

Kinematics of the secondary latch naturally (but not uniquely) decomposes into four 'primitive' kinematic functions described below and illustrated in Figures 2(a)-(d):

1. When the striker impacts surface $A$, the resulting contact force pivots and engages the latch. Assuming adequate striker speed and an appropriate spring constant, the striker then hits surface B; in this case the corresponding contact force will act as an additional reaction force. This force will tend to rotate the striker back to its original position and will ensure that the latch can operate even if the reaction spring is broken. Surface A needs to be curved and convex; its minimum length is determined by the specified allowable misalignment, but its exact value is not critical. Surface B must possess a steep slope towards the bottom of the 'slot'. The steeper slope decreases the probability of accidental release in the event of a broken spring. But once again, no critical dimensions are associated with surface B. 


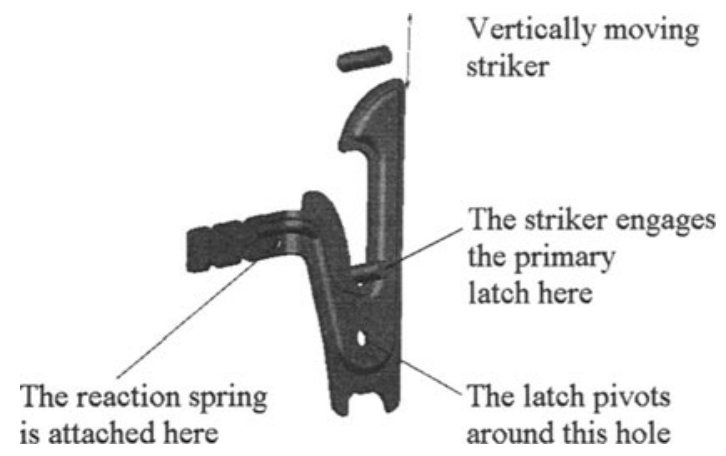

Figure 1 Solid model of a secondary hood latch

2. The second kinematic function is to prevent accidental release and is enforced by surface C. When surface $\mathrm{C}$ is in contact with the striker, the resulting reaction force should not rotate the secondary latch. This requirement translates into sign conditions on the slope of surface $\mathrm{C}$, but its precise shape is somewhat arbitrary.

3. The pivoting motion of the latch is necessary to release the striker. The range of the pivoting motion is controlled by the linear surface $\mathrm{D}$. The controlling parameter is the maximum angle between $\mathrm{D}$ and the vertical axis. It should be clear that the pivoting motion can be accommodated by a wide range of such angles.

4. The last kinematic feature of the latch also has to do with a possible failure mode. Suppose the striker did not engage the primary latch, and the spring is broken; then the striker is free to move vertically, while the secondary latch is free to rotate. If surface $\mathrm{E}$ were convex, its contact with the striker would force the secondary latch to rotate and possibly release the striker. Therefore, surface $E$ can be either concave or straight.

\subsection{Strength considerations}

There are two strength requirements on the latch. The first requirement is that the latch has to withstand the impact of the striker at contact as illustrated in Figure 2(a). The applied load is relatively small and this requirement is trivially satisfied. The second requirement is associated with the kinematic condition in Figure 2(b) and is much more stringent. If the primary latch is not fully engaged and the automobile is moving at high speed, the striker (and therefore the hood) moves upwards - producing dynamic loads 


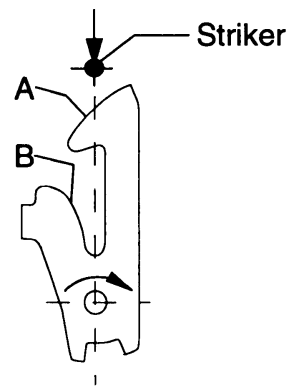

(a) kinematics 1

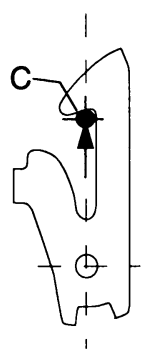

(b) kinematics 2

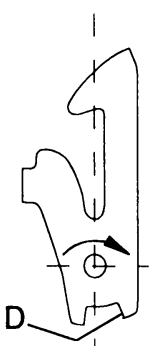

(c) kinematics 3

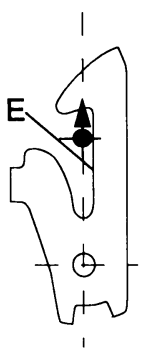

(d) kinematics 4

Figure 2 Four kinematic functions of the latch

in thousands of newtons. These considerations immediately define relevant surfaces where boundary conditions can be applied, but its effect on the rest of the latch's geometry is difficult to analyze for several reasons:

- The shown design implies a Hertzian contact (with high contact forces), but the precise boundary conditions are not known;

- Linear elastic finite element analysis may not be sufficient, and the latch may have to be analyzed for buckling;

- Any such analysis may produce binary safe/fail answers that do not yield an insight in how the shape may be modified to improve strength characteristics.

In practical terms, this leads to an iterative design by analysis and/or physical testing, which tends to be inefficient and sometimes incorrect. The latch shown in Figure 1 has been reinforced by a depression, but the exact form of the depression is not well understood.

\subsection{Spatial Containment}

A properly functioning latch must not interfere with the neighboring assemblies but, as it is typical for such parts, the exact packaging constraints have not been represented and may not even be known. However, it seems intuitively clear (and could be formally stated) that only portions of the latch that come in stable contact with other parts may require tight dimensioning and tolerancing. In this particular case, the circular hole where the latch is attached to the rest of the assembly is the only such feature.

The spatial containment of the moving latch can be studied by 'sweeping' the latch along the trajectory of the motion and intersecting the resulting swept volume with other neighboring parts and/or assemblies. As is the case with the strength analysis, such interference detection computations produce a binary 
safe/fail answer without identifying the remedy. In this particular case, the packaging constraint was encoded in the angular dimension and its tolerance on surface D in Figure 2(c).

\subsection{Manufacturing considerations}

Process dependent manufacturing considerations deal with tolerance control, number of steps in the manufacturing process, form of depression of the latch, difficulty in obtaining the specified shape, material savings and other cost-related factors. The importance of these factors varies and is often determined by the lot size. For example, as the lot size increases, so does the importance of material savings, while the cost of custom tooling becomes less significant. Because automotive latches are produced in large quantities, little (if any) of their geometry is determined by manufacturing consideration - apart from the requirement of being 'producible'.

\section{REENGINEERING THE DESIGN PROCESS}

\subsection{Iterative design using complete geometric information}

In a typical design scenario, functional and manufacturing characteristics of a part are considered by a team of product engineers and are embedded into informal drawing of the resulting shape together with notes on its key features and tolerances. This informal model is then turned into a solid model of the part, and later into a completely annotated engineering drawing; both the solid model and the drawing are supposed to be 'informationally complete'. But the functional and manufacturing characteristics themselves are not part of the created model.

Mechanical designs rarely stay unchanged for a long period of time. The initial designs are never quite correct and they undergo numerous changes before being put into production. Changes in customer specifications, relationships with suppliers, engineering improvements, product failures, and economics of manufacturing are few of the many reasons why mechanical designs are constantly being modified.

It is during such modifications we discover that 'informationally complete' geometric models do not have much of the required information. Suppose we discover (either by physical testing or computer simulation) that the latch fails, and its shape must be modified. Which portions of the latch's shape can be modified, while preserving its functionality? The original design team possessed the needed knowledge and would be able to answer this question; but such information is not explicitly captured by the geometric model, and the current process relies on human knowledge and product history documented elsewhere.

An iterative and inefficient production process is the natural consequence of the above approach to managing engineering information. Geometry of the part is modified as needed to meet the current requirements; then the modified design is sent again through the entire design cycle to make sure that no other requirements have been violated. A 'concurrent engineering' model attempts to 'localize' such iterations, but they cannot be eliminated using geometric information alone. After several such iterations, determining the purpose of various form elements becomes very difficult, if not impossible. Variant designs that have evolved over a number of years typically contain unnecessary geometric details whose rationale is simply no longer known. Furthermore, difficulties in maintaining complete geometric or solid information on a part throughout its life cycle slow the process even further and often lead to inconsistencies between various geometric models, recorded engineering information, and physical artifacts. Such discrepancies 
are easily detected in the example solid model of the latch and the two-dimensional profiles that were obtained from (supposedly) corresponding engineering drawings.

\subsection{An alternative design model}

An alternative and more rational approach to design may be possible, if all engineering decisions related to functionality and manufacturing were recorded and explicitly related to the geometric information they impact. Figure 3 shows how an initial shape of the latch could have been designed starting with a simple rectangular envelope (packaging constraint) and then modifying its shape to satisfy its kinematic requirements one at a time. Every time a new geometric detail is created or modified, it should also contain a pointer to the engineering information that caused the action. Figure 3(d) shows the partially defined geometry of a latch that satisfies all kinematic requirements of the latch.

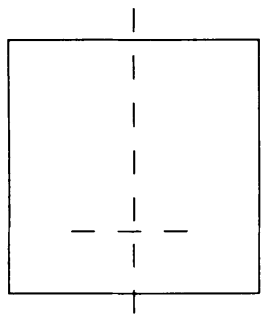

(a) INITIAL PACKAGING CONSTRAINTS

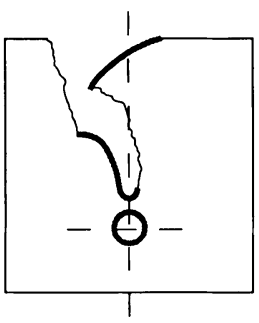

(b) STRIKER GOES DOWN (kinematics 1)

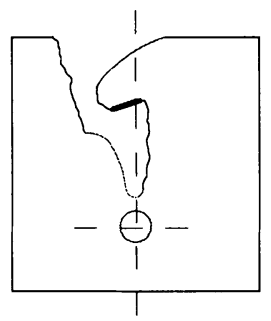

(c) STRIKER SHOULD BE STOPPED (kinematics 2)

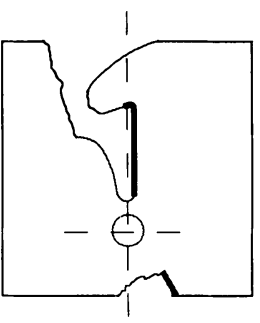

(d) RELEASING THE STRIKER (kinematics 3 and 4 )

Figure 3 A step by step design of the latch kinematics related form

Note that much of the geometry in Figure 3(d) is arbitrary in the sense that it has not been modified from the initial state by kinematic requirements. Additional geometric details are added by considering other functional requirements, such as loading and mating conditions. For the secondary latch, spatial containment, strength characteristics, and manufacturing considerations serve as constraints on the shape of the latch, but they do not define all geometry precisely.

Combining all functional requirements could be captured in a single model that may only partially define geometry of the part and specifies a number of constraints on how the rest of the geometry may vary. In other words, the result of such a design process is a specification for a class of parts that satisfy the combined model. Figure 4 shows what such a model might be. Partially specified geometries are linked together in a single combinatorial (graph-like) structure that also serves as a carrier for relevant physical quantities, such as forces, temperatures, and displacements. Chain models proposed in (Palmer \& Shapiro 1994) may be used to formally define semantics of such models. Some constraints may be expressed in terms of parameters of the combinatorial model; others, such as packaging constraints, may require quite different set-theoretic means such as set operations, sweeping, and Minkowski operations. 
Today we do not have a formal definition for such a model, but it seems apparent that such a definition can be constructed.
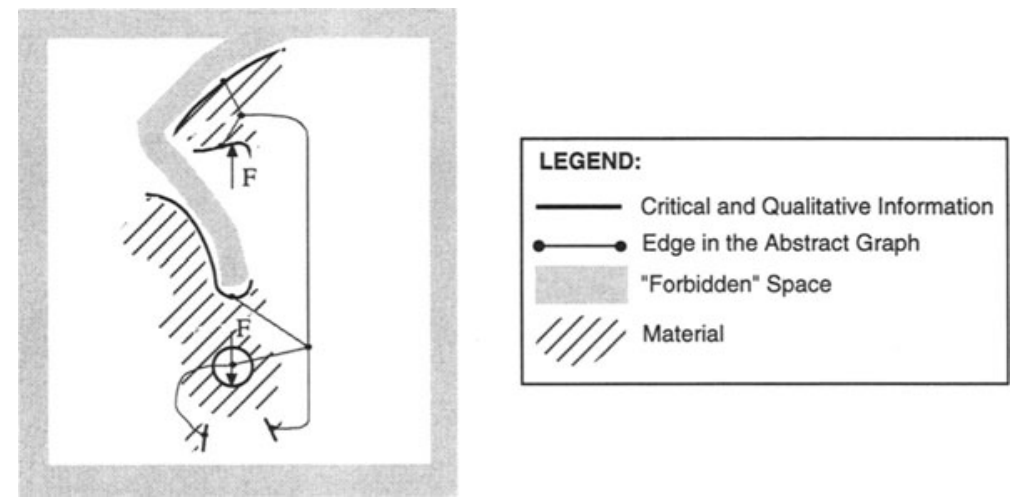

Figure $4 \mathrm{~A}$ model of the latch should include combinatorial, physical, and geometric information

\subsection{Benefit: systematic maintenance of designs}

We argued above that the current practice of communicating all engineering information through geometry does not adequately support systematic, rational, and efficient design process. A more appropriate model, such as described above, would contain explicit links between geometry and engineering (functional and manufacturing) characteristics. This would allow identifying critical and non-critical shape parameters, enable systematic modification, and minimize costly iterations. We now illustrate some of these possibilities using once again the secondary latch as the example.

- Geometry of surface $\mathrm{C}$ in Figure 2(b) is determined solely by its interaction with the striker. It's current shape results in a Hertzian contact between the striker and the latch, causing high stress concentration. Changing shape of surface $\mathrm{C}$ as shown in Figure 5(a) would increase contact area between the striker and the latch, thus decreasing the stresses - without compromising functionality of the latch. Increase in the contact area may be also achieved by modifying the shape of the striker.

- In the current design, surface D (see Figure 2(c)) is tightly toleranced; the above analysis reveals that its only purpose is to control the range of angular motion in order to satisfy a packaging constraint. However, a smaller angle would not violate the assumed packaging constraint or intended kinematic functionality of the latch and would eliminate the (otherwise critical) tolerance.

- Careful analysis of surface B in Figure 2(a) reveals that the same functionality may be achieved with a simpler surface (shown in Figure 5(b)) and lead to savings in material. 
- The original shape of the latch contains a number of unnecessary complex elements that are a byproduct of the current design process. Based on our model above, we can simplify the shape as shown in Figure 5(b) without affecting its functionality, leading to a more effective manufacturing process.

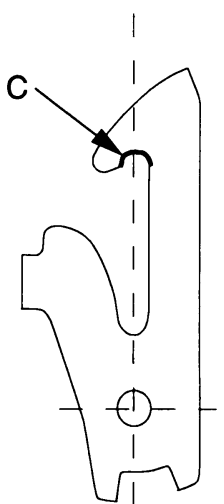

(a) Modifying the shape of surface $C$ increases the contact area and reduces stresses

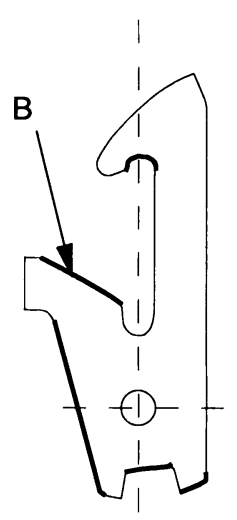

(b) Simpler geometry doesn't compromise functionality of the latch

Figure 5 Systematic analysis suggests possible improvements in latch design

We do not claim that these particular changes in the design of the latch are major improvements. But we do maintain that such product analysis and systematic modifications are possible only when models of mechanical products such as described above become available; such activities are not properly supported by the current product design process based on communicating with geometric and solid models.

\section{CONCLUSIONS}

\subsection{Legacy of geometry-based systems}

Effectiveness of geometry-based systems is rooted in human ability to use geometric descriptions for communicating engineering information. In principle, geometry of an idealized physical artifact can be described completely and unambiguously, and therein lies the main strength of solid modeling. There is little doubt that solid modeling revolutionized the way in which geometric information is created 
and maintained throughout the manufacturing enterprise. However, the theoretical spatial informational completeness of solid modeling does not translate into 'engineering informational completeness.' The latter is a myth for a number of reasons:

Today solid models cannot represent all geometric information 'exactly', because a number of difficult issues, for example those related to numerical robustness and semantics of mechanical tolerances, have not been resolved. Ironically, the single most important application of solid modeling so far has been in expediting production and managing of engineering drawings, which are often further processed and annotated with additional textual information.

- Even if we could represent 'exactly' all of the geometric information using solid models, we may not want to do so. Since functional information does not define geometry completely, it may be advantageous to leave some geometric detail to be determined by the manufacturing process. Engineering drawings in a vertically integrated company often do contain informal notes such as 'typical' or 'as formed' that are related to specific manufacturing processes. A considerable freedom in geometry of mechanical parts has been observed in a different context in (Samuel et al 1976).

- Requirement of 'exact' geometric information translates into a significantly higher burden on those who create this information using solid modeling systems. Knowing that geometric information will be supplemented with additional informal notes and engineering data allows users to get around many difficult problems related to blending and filleting, tolerance allocation, careful detailing, and so on.

- Finally, from an engineering point of view, no purely geometric model can be 'informationally complete' because it simply does not contain critical information related to product functionality and manufacturing. Geometric information alone does not form a rational basis for deciding if two products are 'similar,' for modifying and improving existing products, or for adopting new manufacturing methods.

Failure to recognize these limitations of geometric and solid modeling technology may lead to computerizing (and institutionalizing) of inefficient and outdated industrial practices - ultimately resulting in a highly iterative and inefficient enterprise.

\subsection{New models for product development}

Figure 4 is an attempt to describe pictorially the kind of model for a mechanical part that may be more useful in product development. Before such models can become part of commercial systems, they need to be formally defined and understood. We are only beginning to take steps in this direction.

A key notion in constructing a formal model for a mechanical part appears to be its combinatorial structure - a hierarchical graph-based description of parts' functional and manufacturing characteristics in terms of simple, discrete, and interacting 'primitives'. For the latch studied in this paper, the proposed structure appears to be natural, but in general it need not be unique. It is not likely that such a combinatorial structure could be described by a tree, because some geometric elements of the part are likely to be involved in mechanical function sharing (Ulrich 1990). Shapiro and Voelcker (Shapiro \& Voelcker 1989) suggested governing principles for a combinatorial decomposition of mechanical artifacts, and algebraic-topological chain models proposed by Palmer and Shapiro (Palmer \& Shapiro 1993) may provide the tools for formalizing them.

The combinatorial model of a mechanical part appears to be consistent with the principle of process independence, because it contains virtually no manufacturing information. On the other hand, many important characteristics of mechanical parts do not seem to be captured by a pure combinatorial model. 
These include packaging consideration, motion-related properties, and manufacturing constraints, and other essentially geometric properties. More generally, our analysis suggests that there are at least three broad categories of geometric information illustrated in Figure 6:

Critical: tightly toleranced geometric (form, size, location) elements that are typically related to part's performance and mating conditions in an assembly; the only critical geometry on the secondary latch is the round hole which is used in assembly, kinematic, and loading conditions;

Qualitative: general form properties that are relevant to the functionality of the part, but whose size and location may vary within a relatively wide parametric range; these include convexity and curvature related properties of latch surfaces $\mathrm{A}, \mathrm{B}, \mathrm{C}, \mathrm{D}$, and $\mathrm{E}$;

Discretionary: geometric form is largely undetermined and can vary widely within prescribed constraints; this freedom can be used to choose the geometry that is best suited for a particular manufacturing process or used to optimize functional or manufacturing characteristics (such as weight, strength, material utilization, number of manufacturing operations). Other examples and uses of discretionary geometry can be found in (Samuel et al 1976).

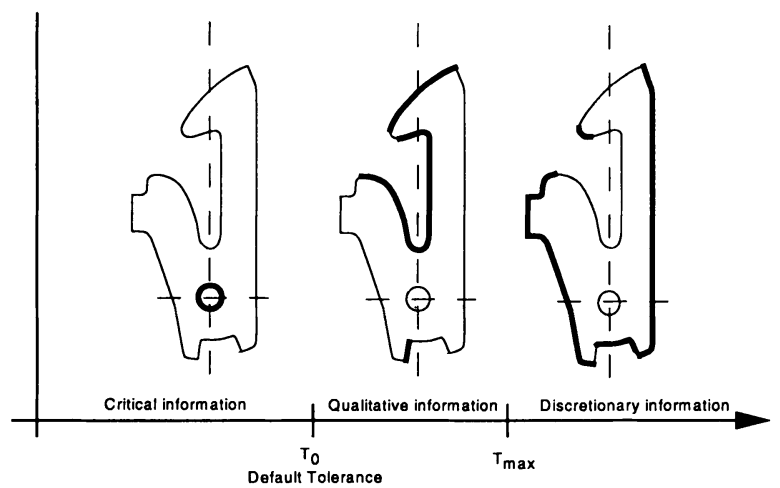

Figure 6 Three categories of geometric information

The dividing lines between the three types of geometry are somewhat blurred but the transition from 'critical' to 'qualitative' clearly depends on the tolerances associated with the precision of the chosen manufacturing process.

In summary, new models of mechanical artifacts are needed to capture explicitly the correspondence between mechanical characteristics and spatial elements. Such models will support systematic product development, efficient modification procedures, and rational tolerancing approaches, thus, eliminating costly errors and iterations in a typical product development cycle. 


\section{ACKNOWLEDGMENTS}

The research described in this paper was started while Vadim Shapiro was a member of research staff at General Motors Research and Development Center in Warren, Michigan and benefited from numerous discussions with Dr. Robert Tilove. The authors are also grateful to Professor Herb Voelcker for reading the earlier version of this paper and suggesting a number of improvements. This work is supported in part the National Science Foundation grants DMI-9502728 and DMI-9522806, by General Motors Corporation, and by EDS Unigraphics.

\section{REFERENCES}

P.J. Booker. A history of engineering drawing. Northgate Publishing, London, 1979.

J. G. Bralla. Handbook of product design for manufacture. McGraw-Hill, Inc., 1986.

National Research Council. Improving Engineering Design: Designing for Competitive Advantage. National Academy Press, Washington, D.C., 1990.

J. J. Cunningham and J. R. Dixon. Designing with features: The origin of features. In ASME Computers in Engineering Conference, San Francisco, CA, 1988.

A. D. Dimaragonas. The origins of engineering design. In ASME DE-Vol. 63, Vibrations of Mechanical Systems and the History of Mechanical Design, Albuquerque, NM, September 19-22 1993.

M. R. Duffey and J. R. Dixon. Automating extrusion design: A case study in geometric and topological reasoning for mechanical design. Computer-Aided Design, 20(10):589-596, 1988.

F. J. Evans and J. J. van Dixhoorn. Towards more physical structure in systems theory. In F. J. Evans and J. J. van Dixhoorn, editors, Physical Structure in Systems Theory, pages 1 - 15, London, 1974. Academic Press.

E.S. Ferguson. Engineering and the Mind's Eye. The MIT Press, 1993.

S. Finger and J. A. Dixon. A review of research in mechanical engineering design. Research in Engineering Design, 1(1 and 2), 1989. Parts I and II.

S. Finger and J. R. Rinderle. A transformational approach to mechanical design using a bond graph grammar. In ASME Design Theory and Methodology Conference, 1989.

E. L. Gursoz, Y. Choi, and F. B. Prinz. Vertex-based representation of non-manifold boundaries. In M. Wozny, J. Turner, and K. Preiss, editors, Geometric Modeling for Product Engineering, pages 107130. North Holland, 1990.

A. Mukherjee and J. Hilibrand, editors. New Paradigms for Manufacturing. National Science Foundation, Washington, D.C., 1994. Workshop held on May 2-4 in Arlington, VA.

R.S. Palmer and V Shapiro. Chain models of physical behavior for engineering analysis and design. Research in Engineering Design, 5:161-184, 1993. Invited paper for the special issue Advances in Representation and Reasoning for Mechanical CAD.

Henry M. Paynter. Analysis and Design of Engineering Systems. The M.I.T. Press, Cambridge, Massachusetts, 1961.

T. Peters, D. Rosen, and V. Shapiro. A topological model of limitations in design for manufacturing. Research in Engineering Design, 6(4):223-233, 1994.

C. Poli and S. Shanmugasundaram. Design for die casting - a group technology based approach. In ASME Design Theory and Methodology Conference, pages 135-141, 1991.

M. J. Pratt. Synthesis of an optimal approach to form feature modelling. In ASME Computers in Engineering Conference, pages 263-274, San Francisco, CA, 1988. 
A. A. G. Requicha and H. B. Voelcker. An introduction to geometric modeling and its applications in mechanical design and production. In J. T. Tou, editor, Advances in Information Systems Science, Vol. 8. Plenum Publishing, 1981.

J. R. Rinderle. Implications of function-form-fabrication relations on design decomposition strategies. In ASME Computers in Engineering, 1986.

J. R. Rossignac. Issues on feature-based editing and interrogation of solid models. Computers and Graphhics, 14(2):149-172, 1990.

J. R. Rossignac and M. A. O'Connor. SGC: a dimension-independent model for pointsets with internal structures and incomplete boundaries. In M. Wozny, J. Turner, and K. Preiss, editors, Geometric Modeling for Product Engineering, pages 145-180. North Holland, 1990.

N. M. Samuel, A. A. G. Requicha and S. A. Elkind. Methodology and Results of an Industrial Part Survey. Tech. Memo 21, Production Automation Project, University of Rochester, Rochester, NY, July 1976.

J. J. Shah. Synthesis of initial form for structural shape optimization. Transaction of ASME, Journal of Mechanical Design, 110:564-570, October 1988.

J. J. Shah. Conceptual development of form features and feature modelers. Research in Engineering Design, 2:93-108, 1991.

V. Shapiro and H. B. Voelcker. On role of geometry in mechanical design. Research in Engineering Design, 1(1):69-73, 1989.

K. Shimada and D. Gossard. Automated shape generation of components in mechanical assemblies. In ASME Design Automation Conference, Tempe, AZ, September 1992.

R. B. Tilove. Set membership classification: A unified approach to geometric intersection problems. IEEE Transactions on Computer, C-29(10):874-883, October 1980.

K. T. Ulrich and W. P. Seering. Synthesis of schematic descriptions in mechanical design. Research in Engineering Design, 1(1):3 - 18, 1989.

K. T. Ulrich and W. P. Seering. Function sharing in mechanical design. Design Studies, 11(4):223 - 234, October 1990.

H. B. Voelcker. Modeling in the design process. In W. Dale Compton, editor, Design and Analysis of Integrated Manufacturing Systems. National Academy Press, Washington, D.C., 1988.

K. J. Weiler. Topological Structures for Geometric Modeling. PhD thesis, Rensselaer Polytechnic Institute, 1986.

ANSI Standard Y14.5M. Dimensioning and Tolerancing. American Society of Mechanical Engineers, New York, NY, 1982. 